\title{
Application of Commensurability in Earthquake Prediction
}

\author{
Youjin $\mathrm{Su}^{1}$, Hui $\mathrm{Hu}^{2}$ \\ ${ }^{1}$ Seismological Bureau of Yunnan Province, Kunming, China \\ ${ }^{2}$ Yunnan Observatory, Chinese Academy of Sciences, Kunming, China \\ Email: suyoujin0818@sina.com, huhui@mail.ynao.ac.cn
}

Received 2 May 2015; accepted 22 June 2015; published 25 June 2015

Copyright (C) 2015 by authors and Scientific Research Publishing Inc.

This work is licensed under the Creative Commons Attribution International License (CC BY).

http://creativecommons.org/licenses/by/4.0/

(c) (i) Open Access

\section{Abstract}

This article introduces application of the expanding commensurability in earthquake prediction. The results show that most of the world's major earthquake occurred at their commensurable points of time axis. An EQ 7.0 occurred in Lushan of China on 2013-04-20 and an EQ 8.2 occurred in Iquique of northern Chile on 2014-04-01 all occurred at their commensurable points of time axis. This once again proves that the commensurability provides an important scientific basis for the prediction of major earthquakes, which will occur in the area in future.

\section{Keywords}

Commensurability, Orders in the Natural World, Earthquake Prediction

\section{Introduction}

Earthquake (EQ) research, especially EQ prediction, is accepted as a worldwide difficult scientific problem [1]. People have made arduous and unremitting efforts for searching for the effective methods of EQ prediction for a very long time. However, most of the basic concepts and systems of methods do not break away from the system of quantitative analysis of the inertial system so that the problem of prediction of serious natural disasters like EQs has not been solved. A Chinese famous geophysicist, Weng Wenbo (1912-1994) studies the Titius-Bode law [2] and firstly pointed out that the occurrence time of EQ has the commensurability and applied the principle of the commensurability which was firstly proposed in astronomy to the prediction of major natural disasters such as earthquakes, droughts and water-loggings, etc., thereby developed it into the theory of prediction which have been widely used in the prediction of disasters [3]-[7]. Based on this theory, the occurrence of a few EQs, droughts and water-loggings, and other natural disasters were successfully predicted. 


\section{Commensurability and Its Expansion}

Commensurability of the word was first proposed by the German famous astronomer Titius who found and proposed it in the study of the average distance between the planets within the solar system to the sun. Later, another famous German astronomer Bode made further studies. This is the famous Titius-Bode Law. From TitiusBode law the distance of the planet $\mathrm{n}$ from the sun can be expressed as:

$$
a_{n}=0.4+0.32 \times 2^{n-2},
$$

It also can be written as the following form:

$$
\beta=\frac{a_{n+1}}{a_{n}},
$$

where $a_{n}$ is the distance of the planet $\mathrm{n}$ to the sun, reckoned in astronomical unit, and $n$ is the number of the planets away from the sun to the far side. For Mercury, the number $n$ is not 1 , instead it is taken as $-\infty$. $\beta$ is the commensurable value for the planets in the solar system [8].

Just as the famous geophysicist Weng Wenbo [9] pointed out that the commensurability is one of the orders in the natural world. The Equation (2) itself brings to light the distribution law of the matter in a space region, and for time domain the commensurability can be expressed as:

$$
\Delta X=\frac{X_{i+\Delta i}-X_{i}}{K}
$$

Here $k$ is an integer. If the above relation is tenable, then the data set $\left\{X_{i}\right\}$ is commensurable. $\Delta X$ is the commensurable value of the data set $\left\{X_{i}\right\}$ and $X_{i}$ and $X_{i+\Delta i} \in\left\{X_{i}\right\}$. If k is equal to 1 , then $\Delta X$ is the period of $\left\{X_{i}\right\} \quad[10]$.

\section{Prediction Practice}

\subsection{Forecast by Weng Wenbo on the EQ Ms7.4 in California of United States on June 28, 1992}

Before 1992 Weng Wenbo made a few forecasts of EQs occurring in United States, most of these forecasts corresponded quite well with the later occurred actual events [11]. As example, on January 17, 1992, according to a request from Prof. Cecil H. Green, Director of American Geophysical Unit, and upon obtaining ratification from senior authorities, Weng Wenbo presented his forecast report to Prof. Green [12];

Time: June 191992

Magnitude: 6.8

Place: Broad region of San Francisco

The predicted EQ occurred on June 28, 1992. Its magnitude Ms7.4, was only Ms0.6 greater in magnitude, and the occurrence date was only 9 days later than the date stated by the forecast report of Weng Wenbo [13].

\subsection{Prediction on the Wenchuan EQ 8.0 and Lushan EQ 7.0 in China and the Iquique EQ 8.2 in Chile of 2014}

The Wenchuan EQ Ms8.0 occurred in Sichuan province of China on May 12, 2008. It caused greatest heavy losses of the life and property in China in recent history. Before the EQ, Long Xiaoxia et al. based on the commensurability using the following commensurable equations with five elements rather precisely predicted that a strong EQ might occur in 2008 [14]:

$$
\begin{aligned}
& X_{23}+X_{15}+X_{1}-X_{3}-X_{7}=2008 \\
& X_{23}+X_{20}+X_{12}-X_{18}-X_{7}=2008 \\
& X_{24}+X_{21}+X_{8}-X_{18}-X_{6}=2008 \\
& X_{25}+X_{15}+X_{7}-X_{19}-X_{3}=2008 \\
& X_{22}+X_{15}+X_{5}-X_{13}-X_{3}=2008 \\
& X_{21}+X_{11}+X_{9}-X_{13}-X_{1}=2008
\end{aligned}
$$


The EQ catalogue used by Long Xiaoxia et al. is given in Table 1. From Table 1 the commensurable value of the EQs occurrence is equal to 2.44 years. Taking $k=5$ we obtain:

$$
2008-05-12=1996.09+2.44 \times 5=2008^{\mathrm{y}} 4^{\mathrm{m}} 15^{\mathrm{d}}+27 \text { days }
$$

Its error which is the opposite of the occurrence date of the earthquake is 27 days. Its relative error is 0.03 .

An EQ 7.0 occurred in Lushan of Sichuan province, China on 2013-04-20. Similar, on the basis of the Table 1 we obtain:

$$
\text { 2013-04-20 }=2008.36+2.44 \times 2=2013^{\mathrm{y}} 3^{\mathrm{m}} 29^{\mathrm{d}}+22 \text { days }
$$

It occurred just at the commensurable point equal to 2 times of its time axis. Its absolute error is 22 days. Its relative error also is 0.03 .

An EQ 8.2 in Iquique, northern Chile, occurred on 2014-04-01. By the research of Engdahl and Villasenor [15] the catalogues of EQs of $M \geq 7.0$ have been complete and reliable since 1900. Therefore we have analyzed the EQs of $M \geq 7.0$ in Chile since 1900.0, and found its commensurable value is 0.59 years (see Table 2). So,

$$
\text { 2004-04-01 }=2010.15+0.59 \times 7=2014^{\mathrm{y}} 04^{\mathrm{m}} 12^{\mathrm{d}}-11 \text { days }
$$

It occurred just at the commensurable point equal to 7 times of its time axis. Its absolute error is 11 days. Its relative error is 0.05 .

On the basis of many years' research for commensurability according to the commensurable principle, we compiled a Fortran program for commensurable analyses of the data. By means of the Fortran program our ana-

\begin{tabular}{|c|c|c|c|c|c|c|}
\hline & Earthquakes date & $X_{\mathrm{i}}-X_{i_{-}}$ & & & $K \Delta X$ & $X_{i}-K \Delta X$ \\
\hline No. & YMD & (year) & (year) & K & (year) & (year) \\
\hline 1 & 19131221 & 1913.97 & & & & \\
\hline 2 & 19170731 & 1917.57 & 3.60 & 1 & 2.44 & 1.16 \\
\hline 3 & 19230324 & 1923.22 & 5.65 & 2 & 4.88 & 0.77 \\
\hline 4 & 19250316 & 1925.20 & 1.98 & 1 & 2.44 & -0.46 \\
\hline 5 & 19330825 & 1933.64 & 8.44 & 3 & 7.32 & 1.12 \\
\hline 6 & 19360427 & 1936.32 & 2.68 & 1 & 2.44 & 0.24 \\
\hline 7 & 19410516 & 1941.37 & 5.05 & 2 & 4.88 & 0.17 \\
\hline 8 & 19420201 & 1942.08 & 0.71 & 0 & 0.00 & 0.71 \\
\hline 9 & 19480525 & 1948.40 & 6.32 & 3 & 7.32 & -1.00 \\
\hline 10 & 19500203 & 1950.09 & 1.69 & 1 & 2.44 & -0.75 \\
\hline 11 & 19520930 & 1952.75 & 2.66 & 1 & 2.44 & 0.22 \\
\hline 12 & 19550414 & 1955.28 & 2.53 & 1 & 2.44 & 0.09 \\
\hline 13 & 19601109 & 1960.85 & 5.57 & 2 & 4.88 & 0.69 \\
\hline 14 & 19670830 & 1967.66 & 6.81 & 3 & 7.32 & -0.51 \\
\hline 15 & 19700105 & 1970.01 & 2.35 & 1 & 2.44 & -0.09 \\
\hline 16 & 19710428 & 1971.32 & 1.31 & 1 & 2.44 & -1.13 \\
\hline 17 & 19730206 & 1973.09 & 1.77 & 1 & 2.44 & -0.67 \\
\hline 18 & 19740511 & 1974.35 & 1.26 & 1 & 2.44 & -1.18 \\
\hline 19 & 19760823 & 1976.64 & 2.29 & 1 & 2.44 & -0.15 \\
\hline 20 & 19790315 & 1979.20 & 2.56 & 1 & 2.44 & 0.12 \\
\hline 21 & 19810124 & 1981.06 & 1.86 & 1 & 2.44 & -0.58 \\
\hline 22 & 19881106 & 1988.84 & 7.78 & 3 & 7.32 & 0.46 \\
\hline 23 & 19890425 & 1989.31 & 0.47 & 0 & 0.00 & 0.47 \\
\hline 24 & 19950712 & 1995.52 & 6.21 & 3 & 7.32 & -1.11 \\
\hline 25 & 19960203 & 1996.09 & 0.57 & 0 & 0.00 & 0.57 \\
\hline 26 & 20080512 & 2008.36 & 12.27 & 5 & 12.20 & 0.07 \\
\hline \multicolumn{3}{|c|}{ Commensurable value } & \multicolumn{4}{|c|}{2.44} \\
\hline \multicolumn{3}{|c|}{ Mean } & \multicolumn{4}{|c|}{-0.031} \\
\hline \multicolumn{3}{|c|}{ Standard deviation $\left(\sigma_{n-1}\right)$} & \multicolumn{4}{|c|}{0.717} \\
\hline
\end{tabular}
lyzed results have been all given in the tables. The two tables all are directly output results by computer.

Table 1. Commensurability of earthquakes in Sichuan-Yunnan region since 1900.0. 
Table 2. Commensurability of EQs in Chile since 1900.0.

\begin{tabular}{|c|c|c|c|c|c|c|}
\hline & Earthquak & $X_{\mathrm{i}}-X$ & & & & \\
\hline No. & YMD & (year) & (year) & $\mathrm{K}$ & (year) & (year) \\
\hline 1 & 19040319 & 1904.21 & & & & \\
\hline 2 & 19060817 & 1906.62 & 2.41 & 4. & 2.36 & 0.05 \\
\hline 3 & 19060830 & 1906.66 & 0.04 & 0. & 0.00 & 0.04 \\
\hline 4 & 19061226 & 1906.98 & 0.32 & 1. & 0.59 & -0.27 \\
\hline 5 & 19090608 & 1909.43 & 2.45 & 4. & 2.36 & 0.09 \\
\hline 6 & 19100906 & 1910.67 & 1.24 & 2. & 1.18 & 0.06 \\
\hline 7 & 19110915 & 1911.70 & 1.03 & 2. & 1.18 & -0.15 \\
\hline 8 & 19140130 & 1914.07 & 2.37 & 4. & 2.36 & 0.01 \\
\hline 9 & 19180520 & 1918.38 & 4.31 & 7. & 4.13 & 0.18 \\
\hline 10 & 19181204 & 1918.92 & 0.54 & 1. & 0.59 & -0.05 \\
\hline 11 & 19221107 & 1922.85 & 3.93 & 7. & 4.13 & -0.20 \\
\hline 12 & 19221111 & 1922.86 & 0.01 & 0 . & 0.00 & 0.01 \\
\hline 13 & 19250515 & 1925.36 & 2.50 & 4. & 2.36 & 0.14 \\
\hline 14 & 19270414 & 1927.28 & 1.92 & 3. & 1.77 & 0.15 \\
\hline 15 & 19281201 & 1928.92 & 1.64 & 3. & 1.77 & -0.13 \\
\hline 16 & 19310318 & 1931.20 & 2.28 & 4. & 2.36 & -0.08 \\
\hline 17 & 19330223 & 1933.14 & 1.94 & 3. & 1.77 & 0.17 \\
\hline 18 & 19360713 & 1936.53 & 3.39 & 6. & 3.54 & -0.15 \\
\hline 19 & 19390125 & 1939.06 & 2.53 & 4. & 2.36 & 0.17 \\
\hline 20 & 19390418 & 1939.29 & 0.23 & 0 . & 0.00 & 0.23 \\
\hline 21 & 19401004 & 1940.76 & 1.4 & 2. & 1.18 & 0.29 \\
\hline 22 & 19420708 & 1942.51 & 1.75 & 3. & 1.77 & -0.02 \\
\hline 23 & 19430314 & 1943.20 & 0.69 & 1. & 0.59 & 0.10 \\
\hline 24 & 19430406 & 1943.26 & 0.06 & 0 . & 0.00 & 0.06 \\
\hline 25 & 19450913 & 1945.70 & 2.44 & 4. & 2.36 & 0.08 \\
\hline 26 & 19460802 & 1946.58 & 0.88 & 1. & 0.59 & 0.29 \\
\hline 27 & 19490420 & 1949.29 & 2.71 & 5. & 2.95 & -0.24 \\
\hline 28 & 19490425 & 1949.31 & 0.02 & 0. & 0.00 & 0.02 \\
\hline 29 & 19530506 & 1953.34 & 4.03 & 7. & 4.13 & -0.10 \\
\hline 30 & 19600521 & 1960.38 & 7.04 & 12. & 7.08 & -0.04 \\
\hline 31 & 19600522 & 1960.39 & 0.01 & 0. & 0.00 & 0.01 \\
\hline 32 & 19600522 & 1960.39 & 0.00 & 0 . & 0.00 & 0.00 \\
\hline 33 & 19600620 & 1960.46 & 0.07 & 0. & 0.00 & 0.07 \\
\hline 34 & 19601202 & 1960.92 & 0.46 & 1. & 0.59 & -0.13 \\
\hline 35 & 19620214 & 1962.12 & 1.20 & 2. & 1.18 & 0.02 \\
\hline 36 & 19650328 & 1965.23 & 3.11 & 5. & 2.95 & 0.16 \\
\hline 37 & 19661228 & 1966.98 & 1.75 & 3. & 1.77 & -0.02 \\
\hline 38 & 19671221 & 1967.97 & 0.99 & 2. & 1.18 & -0.19 \\
\hline 39 & 19710709 & 1971.51 & 3.54 & 6. & 3.54 & 0.00 \\
\hline 40 & 19740818 & 1974.62 & 3.11 & 5. & 2.95 & 0.16 \\
\hline 41 & 19750510 & 1975.35 & 0.73 & 1. & 0.59 & 0.14 \\
\hline 42 & 19811016 & 1981.79 & 6.44 & 11. & 6.49 & -0.05 \\
\hline 43 & 19811107 & 1981.85 & 0.06 & 0. & 0.00 & 0.06 \\
\hline 44 & 19831004 & 1983.75 & 1.90 & 3. & 1.77 & 0.13 \\
\hline 45 & 19850303 & 1985.16 & 1.41 & 2. & 1.18 & 0.23 \\
\hline 46 & 19850304 & 1985.17 & 0.01 & 0. & 0.00 & 0.01 \\
\hline 47 & 19850409 & 1985.26 & 0.09 & 0 . & 0.00 & 0.09 \\
\hline 48 & 19870305 & 1987.17 & 1.91 & 3. & 1.77 & 0.14 \\
\hline 49 & 19870305 & 1987.17 & 0.00 & 0. & 0.00 & 0.00 \\
\hline 50 & 19870808 & 1987.60 & 0.43 & 1. & 0.59 & -0.16 \\
\hline 51 & 19880119 & 1988.05 & 0.45 & 1. & 0.59 & -0.14 \\
\hline 52 & 19880205 & 1988.09 & 0.04 & 0. & 0.00 & 0.04 \\
\hline 53 & 19950730 & 1995.57 & 7.48 & 13. & 7.67 & -0.19 \\
\hline 54 & 19971015 & 1997.78 & 2.21 & 4. & 2.36 & -0.15 \\
\hline 55 & 19980130 & 1998.07 & 0.29 & 0. & 0.00 & 0.29 \\
\hline 56 & 20050613 & 2005.44 & 7.37 & 12. & 7.08 & 0.29 \\
\hline 57 & 20100227 & 2010.15 & 4.71 & 8. & 4.72 & -0.01 \\
\hline \multicolumn{4}{|c|}{ Commensurable value } & \multicolumn{2}{|c|}{0.590} & \\
\hline \multicolumn{4}{|c|}{ Mean } & \multicolumn{2}{|c|}{-0.027} & \\
\hline \multicolumn{4}{|c|}{ Standard deviation $\left(\sigma_{n-1}\right)$} & \multicolumn{2}{|c|}{0.142} & \\
\hline
\end{tabular}


From Table 1 and Table 2 the results show that the EQs basically all occur at the commensurable point of its time axis, respectively. It also shows that the EQs occurrence is not accidental, and there is its pattern and inevitability, only the commensurable value is different for EQs occurred in different areas.

In the Tables, $\Delta X$ is the commensurable value of the studied region. $K$ means that the EQ lies on $K$ times of the commensurable value $\Delta X$ behind the latest recent EQ occurred in the area in its time axis. In the Tables, $X_{i}-X_{i-1}$ is the time interval between major EQs occurring in the same area. For example, EQ No. 3 in Table $1, X_{3}=1923.22, X_{2}=1917.57$, the time interval between the $3^{\text {rd }}$ and $2^{\text {nd }}$ EQ is 5.65 year (unit: years). The corresponding $\mathrm{K}$ value under EQ No. 3 is $\mathrm{K}_{3}=2$, i.e. the 3rd EQ occurred on the second commensurable point in its time axis after the $2^{\text {nd }} E Q$. According to $K_{3}$ and the commensurable value, the predicting point equals to 1917.57 adding the product of 2 and the commensurable value (i.e. $1917.57+2 \Delta X=1917.57+4.88$ ). Its prediction error (i.e. the difference between the predicted point and actual time of occurrence of the third EQ) is 0.77 years.

\section{Discussion and Conclusion}

1) The commensurability revealed by Titius-Bode itself brings to light the distribution law of the matter in a space region, and the expanding commensurable theory proposed by Weng Wenbo reveals the time law of the occurrence of the events in a specified space region. It can be seen that the commensurability is present in various natural phenomena and has universality. It is helpful to study the complicated relationships among various matters, and thus merits further in-depth research.

2) The occurrence of the events seems to be a random accident. In fact, that is not the case. It is in the accident that the necessity resides. Therefore, the commensurability can provide a scientific basis for the prediction of events which may occur.

3) After commensurable value can be determined, $K$ values should be used in order of $K=1,2,3, \cdots$, i.e. when $K=1$ is used but earthquake does not occur, then use $\mathrm{K}=2, \cdots$. The predicted point extrapolated in the time axis by the commensurable value is only a necessary condition, and therefore certain false forecasts are also inevitable, because the exact occurrence time is determined by multiple complex factors. In order to obtain precise prediction, this method must be used in collaboration with other relevant methods, taking the approach of comprehensive analysis.

\section{Acknowledgements}

We extend heartfelt thanks to the referees for their valuable comments. Dr. Chen I-wan, British (ancestor Chinese), Former Advisor of Committee of Natural Hazard Prediction of China Geophysics Society gave us valuable discussion and help. We express our heartfelt thanks to him.

This work is supported by the tackle key project of the ministry of science and technology of P. R. China (2012BAK19B01).

\section{References}

[1] Geller, R.J., Jackson, D.D., Kagan, Y.Y., et al. (1997) Earthquakes Cannot Be Predicted. Science, 275, 1616-1617. http://dx.doi.org/10.1126/science.275.5306.1616

[2] Nieto, M.M. (1972) The Titius-Bode Law of Planetary Distances, Its History and Theory. Pergamon Press, New York.

[3] Dong, G.S. (1987) Preliminary Study on Commensurability of Strong Earthquakes $(M>8)$ within the Continent of China. Journal of Seismological Research, 10, 441-446.

[4] Men, K.P. (2003) Ordered Network Feature of M > 7 Earthquakes and Its Prediction in Northern Part of QinghaiXizang Plateau. Progress in Geophysics, 18, 765-771.

[5] Hu, H. and Han, Y.B. (2005) Prediction of the Hualian Earthquakes in Taiwan and an Extended Discussion on the Method of Commensurability. Applied Geophysics, 2, 194-196. http://dx.doi.org/10.1007/s11770-005-0023-7

[6] Fan, C.R. and Li, X.B. (2008) Commensurable Network Structural Drawing of Major Drought and Flood in Three Gorges of Changjiang and Bengbu of Huaihe. China Flood \& Drought Management, 2, 41-45.

[7] Lin, H., Su, Y.J., Han, Y.B. and Hu, H. (2009) Commensurability of the Earthquakes of Kunlun Area in Xinjiang. Journal of Natural disasters, 18, 189-191.

[8] Zhang, Y.Z., et al. (1980) Encyclopaedia of China: Astronomy. Encyclopaedia of China Press, Beijing, 375,

[9] Weng, W.B. (1981) Commensurability. Acta Geophysical Sinica, 24, 151-154. 
[10] Weng, W.B. (1984) Fundmentals of Forecasting Theory. Petrochemical Industry Press, Beijing.

[11] Xu, D.Y. (1992) The Prediction of California Earthquakes in USA in 1992-A Lecture Given by Prof. Weng Wen-Bo. Acta Geophysica Sinica, 36, 801. (In Chinese)

[12] Weng, W.B. (1992) Letter of Green Giving to Weng Wenbo (Personal Communicate of Letter). In: Wang, M.T., et al., Eds., Academician Weng Wenbo and His Forecast for Natural Disaster, Petrochemical Industry Press, Beijing, 235.

[13] Green, C.H. (1992) Letter of Weng Wenbo Giving to Green (Personal Communicate of Letter). In: Wang, M.T., et al., Eds., Academician Weng Wenbo and His Forecast for Natural Disaster, Petrochemical Industry Press, Beijing, 233.

[14] Long, X.X., Yan, J.P., Sun, H. and Wang, Z.Z. (2006) Study on Earthquake Tendency in Sichuan-Yunnan Region Based on Commensurability. Journal of Atastrophology, 21, 81-84.

[15] Engdahl, E.R. and Villasenor, A. (2002) Global Seismicity: 1900-1999. In: Lee, H.K., Kanamori, H., Jennings, P.C., et al., Eds., International Handbook of Earthquake and Engineering Seismology, Part A, Academic Press, Amsterdam. 